\title{
Non-invasive assessment of changes in left ventricular relaxation by combined phono-, echo-, and mechanocardiography
}

\author{
M MATTHEOS, E SHAPIRO, P J OLDERSHAW, R SACCHETTI, D G GIBSON \\ From the Department of Cardiology, Brompton Hospital, Fulham Road, London
}

SUMMARY In order to investigate interrelations between early diastolic events, simultaneous apex-, phono-, and echocardiograms were recorded in 10 normal subjects and 55 patients with ischaemic heart disease. In normal subjects isovolumic relaxation time measured as A2 to mitral valve opening was $72 \pm 9 \mathrm{~ms}$, the interval A2-0 point was $116 \pm 15 \mathrm{~ms}$, and the amplitude of the $f$ wave of the apexcardiogram relative to the total amplitude was $11 \pm 2 \%$. In patients with a normal left ventricular end-diastolic pressure ( $<15 \mathrm{mmHg}$ ), isovolumic relaxation time was prolonged to $99 \pm 18 \mathrm{~ms}, \mathrm{A2}-0$ point to $162 \pm 5 \mathrm{~ms}$, and the relative height of the $f$ wave was increased to $21 \pm 4 \%$. Isovolumic relaxation time and A2-0 point interval both showed significant inverse correlation with left ventricular diastolic pressure. Glyceryl trinitrate administration or isometric stress both caused well recognised changes in arterial pressure and cavity size. Neither had any additional effect during early diastole in normal subjects. In the patients, however, glyceryl trinitrate prolonged isovolumic relaxation time, delayed the 0 point of the apexcardiogram with respect to $A 2$, increased the interval 0 point $-\mathrm{f}$ wave, and reduced the relative amplitude of the $f$ wave. Isometric stress had the opposite effects. These changes extend beyond the end of ventricular relaxation and so are more readily explained on a mechanical basis, possibly as oscillations whose periodicity and degree of damping were increased by glyceryl trinitrate and decreased by isometric stress.

Disturbances of relaxation form an important component of left ventricular disease, so that their noninvasive investigation may have appreciable clinical significance. In addition, it is clear that diastolic events can be influenced pharmacologically in a way that cannot be predicted from observations in normal subjects. ${ }^{1}$ In the present study, therefore, we examined the haemodynamic and other determinants of a series of well-defined diastolic time intervals in normal subjects and patients with ischaemic heart disease. It was our purpose to investigate mechanisms underlying resting abnormalities in these patients, and to assess the extent and nature of their alteration with glyceryl trinitrate administration or isometric stress.

\section{Subjects and methods}

We investigated 10 normal subjects and 55 patients with ischaemic heart disease, 16 of whom were on Accepted for publication 11 September 1981 therapeutic doses of beta-blocking drugs at the time of study. All patients had the diagnosis confirmed by cardiac catheterisation and coronary arteriography; in 30 , the investigation was performed within 48 hours of cardiac catheterisation and in those patients, diastolic time intervals were correlated with haemodynamic values.

Non-invasive measurements were made with the patients supine and in the left semilateral position. Simultaneous echo-, phono-, and apexcardiograms were recorded along with standard lead II of the electrocardiogram using Cambridge Instruments equipment. Echocardiograms were recorded at the level of the tips of the mitral valve leaflets, to show the time of separation of anterior and posterior cusps at the start of ventricular filling. Left ventricular cavity size was estimated at this level as the distance between the leading edges of the echoes from the left side of the septum and the endocardium of the posterior wall. Phonocardiograms were recorded from the left sternal edge, using a medium or high frequency filter, in the 
position where A2, the onset of the initial high frequency vibration of the aortic component of the second sound, was most obvious. This was definitively identified either from its relation to the dicrotic notch of the indirect carotid pulse, or from the aortic valve echogram (Fig. 1). Apexcardiograms were recorded using a transducer of time constant 4 seconds and lower frequency limit of $0.05 \mathrm{~Hz}$. Records were made photographically at a paper speed of $100 \mathrm{~mm} / \mathrm{s}$, and digitised. ${ }^{2}$

\section{MEASUREMENTS}

From these records, the following measurements were made:

(1) Time intervals

(a) Isovolumic relaxation, as the interval between A2 and the onset of mitral valve opening (Fig. 1). Measurements of this time interval were made blindly by two independent observers. In each patient results from five successive beats were meaned.

(b) A2 to the 0 point of the apexcardiogram. (c) 0 point to the peak of the $f$ wave of the apexcardiogram (Fig. 2).

(2) Relative amplitude of $f$ wave of apexcardiogram, expressed as a percentage of total amplitude (Fig. 2)

(3) From digitised echocardiograms

(a) End-diastolic (synchronous with the q wave of the electrocardiogram) and minimum cavity dimension.

(b) Peak normalised rate of change of dimension during systole (peak VCF).

(c) Any dimension change during isovolumic relaxation.

(d) The time interval between A2 and minimum dimension.

(e) Delay in mitral valve opening, with respect to minimum dimension.

Contraction pattern was described as synchronous $\mathrm{O}$ when A2 preceded minimum cavity dimension by more than $20 \mathrm{~ms}$, and asynchronous when A2 preceded by less than $20 \mathrm{~ms}$, or was synchronous with minimum dimension. ${ }^{1}$

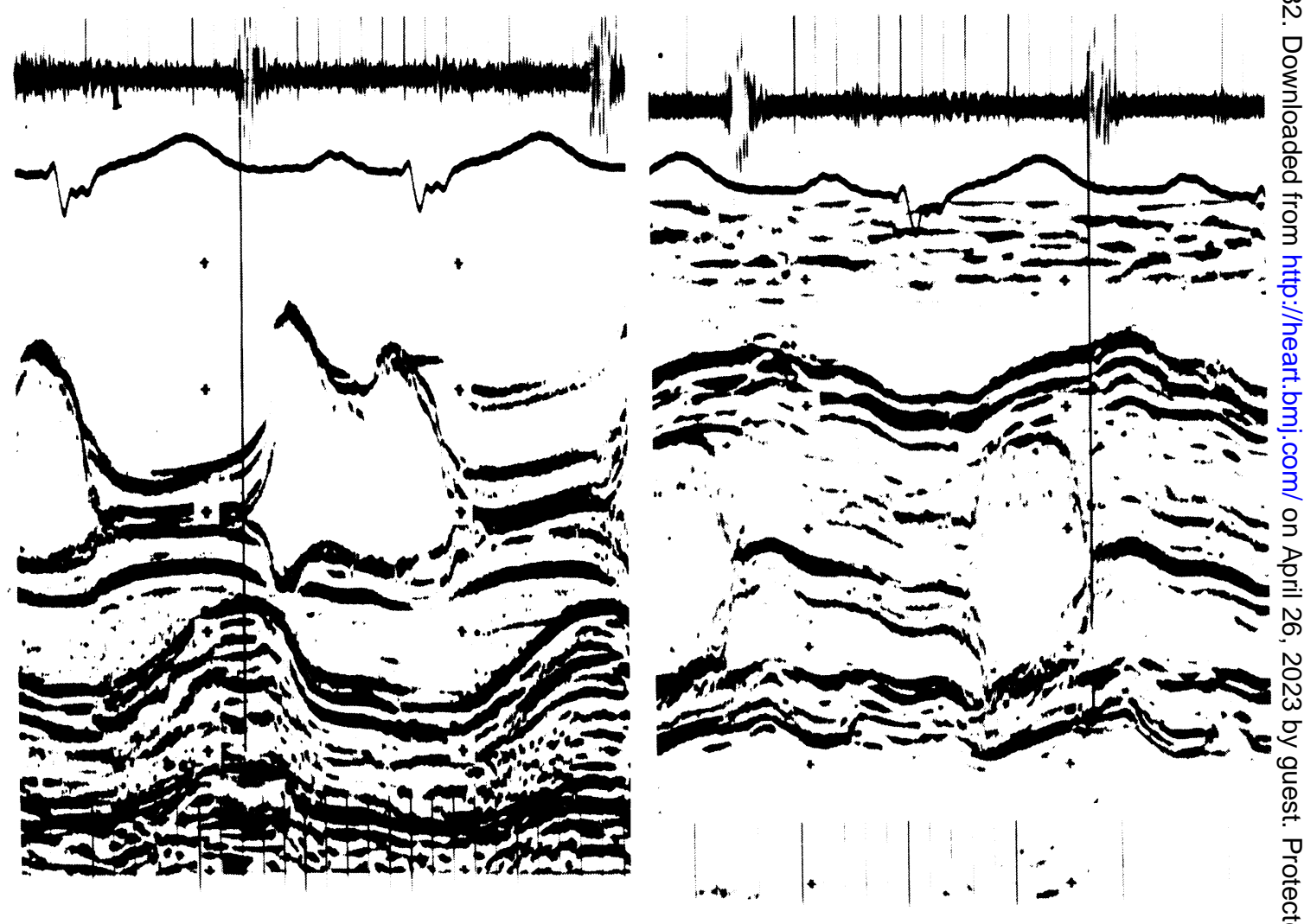

Fig. 1 Measurement of isovolumic relaxation time as the time interval from A2 to mitral valve opening (left panel). The timing of the $\frac{\mathbb{Q}}{\mathrm{Q}}$ onset of the first high frequency component of $A 2$ as aortic valve closure is confirmed from the aortic echogram on the right. 
Thirty patients performed isometric stress when they had completed control measurements. The patients squeezed a sphygmomanometer bulb at $30 \%$ of the maximum force that they could generate for a period of three to five minutes, until the onset of fatigue. Recordings were made during the last $30 \mathrm{sec}-$ onds of effort. No patient developed anginal pain or significant ST segment changes during this time. After 20 minutes' rest, control measurements were repeated, and the patients took two $0.5 \mathrm{mg}$ glyceryl trinitrate tablets sublingually over an interval of five minutes. Measurements were repeated at two, five, ten, and 20 minutes, and those coinciding with the maximum heart rate response were noted.

The statistical significance of differences between mean values was assessed by Student's $t$ test. Linear regression analysis was by the method of least squares. Fourfold tables were analysed by Fisher's exact probability test.

\section{Results}

(1) ISOVOLUMIC RELAXATION TIME

Normal isovolumic relaxation time was $72 \pm 9 \mathrm{~ms}$ (mean \pm 1 standard deviation). In patients with ischaemic heart disease, the mean value was $68 \pm 37 \mathrm{~ms}$, not significantly different. (The mean values for isovolumic relaxation time in normals and patients with ischaemic heart disease for the two observers were $73 \pm 9$ compared with $70 \pm 10$ and $66 \pm 43$ compared with $69 \pm 47$, respectively. The correlation coefficient for the plot of one observer's values against the other observer's values was 0.91 ).

Isovolumic relaxation time was unrelated to peak, diastolic, or mean aortic pressure. There was, however, significant correlation with left ventricular enddiastolic pressure (Fig. 3), given by the equation:

$$
\text { IVR=126-4.0 (pre "a" LVEDP), }
$$

$\mathrm{R}=-0.87=20 \mathrm{~ms}$, where IVR is isovolumic relaxation time and LVEDP is left ventricular enddiastolic pressure. Standard error of the estimate $=20 \mathrm{~ms}$.

In patients with a high left ventricular end-diastolic pressure, therefore, the duration of the isovolumic relaxation time was reduced, in some cases to $0 \mathrm{~ms}$ (Fig. 1). In patients with left ventricular end-diastolic pressure less than $15 \mathrm{mmHg}$ considered in isolation, isovolumic relaxation time was prolonged to $99 \pm 18$ $\mathrm{ms}$, significantly longer than in the normal group $(\mathrm{p}<0.01)$. There was no relation between contraction pattern, whether synchronous or asynchronous, and isovolumic relaxation time.

(2) A2-0 POINT INTERVAL

This interval was $116 \pm 15 \mathrm{~ms}$ in normal subjects,

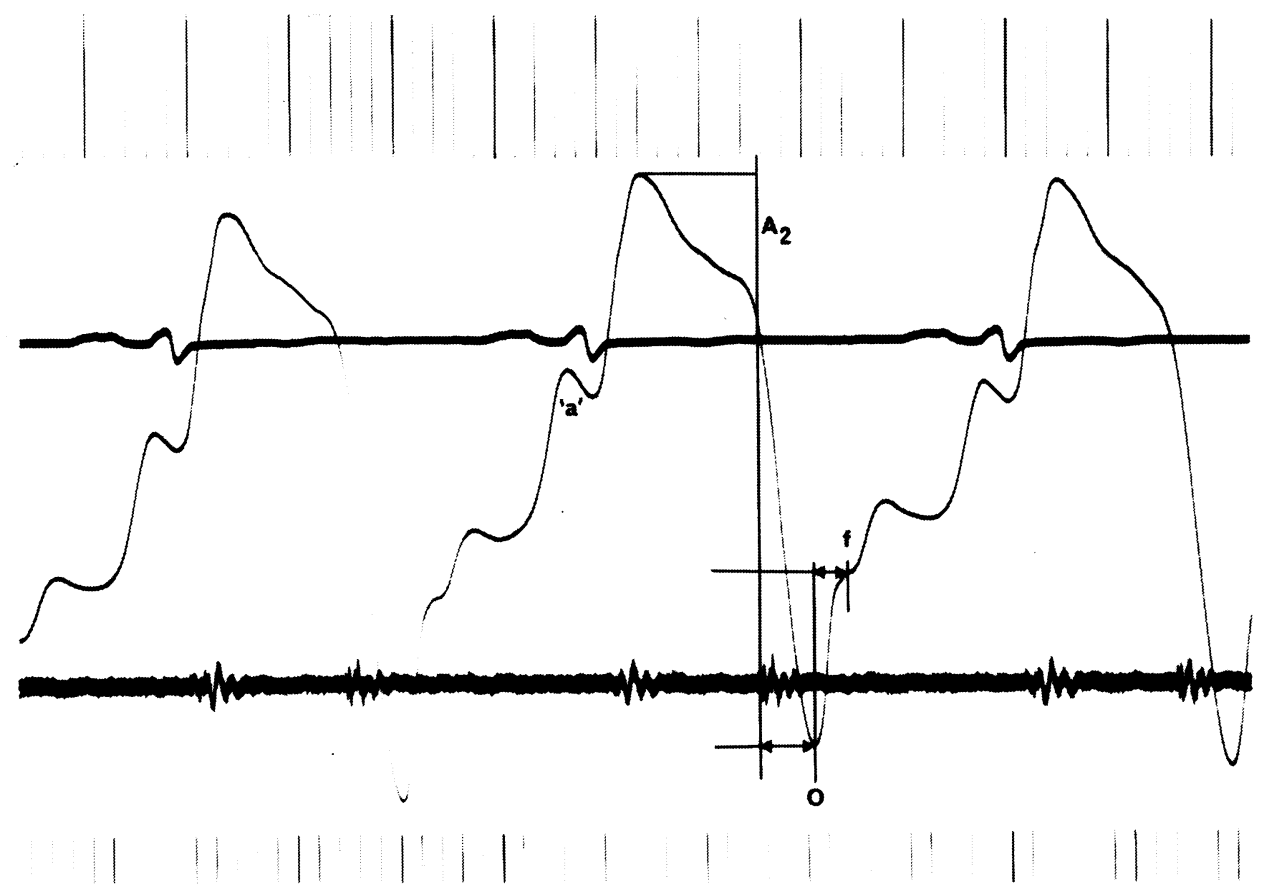

Fig. 2 Representative apexcardiogram, showing measurements of the intervals $A 2-0$ point, $0-f$, and relative amplitude of the $f$ wave. 


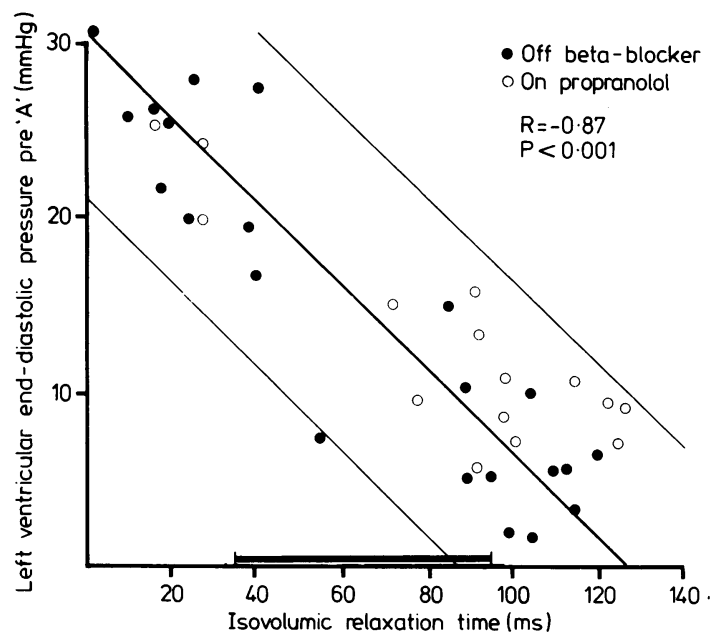

Fig. 3 Relation between isovolumic relaxation time and left ventricular end-diastolic pressure in patients with ischaemic heart disease. The $95 \%$ confidence limits normal for isovolumic relaxation are indicated by the horizontal bar.

significantly longer than isovolumic relaxation time, since the 0 point consistently follows mitral valve opening. Again, in the whole group of patients with ischaemic heart disease, it was not significantly prolonged, being $130 \pm 24 \mathrm{~ms}$. There was, however, strong inverse correlation with end-diastolic pressure, given by the regression equation:

$$
\begin{aligned}
& \text { A2 }-0=175-2.5 \text { (pre "a" LVEDP) } \\
& R=-0.95 \text {, SEE } 23 \mathrm{~ms}
\end{aligned}
$$

for patients not on beta-blockers. For those on betablockers the correlation was significantly less good (Fig. 4).

The resulting relation between end-diastolic pressure and the interval mitral valve opening (MVO) to 0 point is given by the regression equation:

MVO-0 $=44+1.76$ (pre " $a$ " LVEDP), $R=0.77$, $\mathrm{SEE}=13 \mathrm{~ms}$.

The interval A2-0 point in patients with ischaemic heart disease and normal left ventricular end-diastolic pressure was significantly prolonged to $162 \pm 5 \mathrm{~ms}$ $(\mathrm{p}<0.01)$, with respect to normal.

\section{(3) AMPLITUDE AND TIMING OF F WAVE}

In normal subjects the peak of the $f$ wave occurred $75 \pm 10 \mathrm{~ms}$ after the 0 point, and its relative amplitude was $11 \pm 2 \%$. In patients with raised end-diastolic pressure, the relative height of the $f$ wave was significantly increased to $21 \pm 4 \%$. There was also a non-linear relation between the duration of isovolumic relaxation and the relative amplitude of the $f$ wave (Fig. 5), so that as isovolumic relaxation

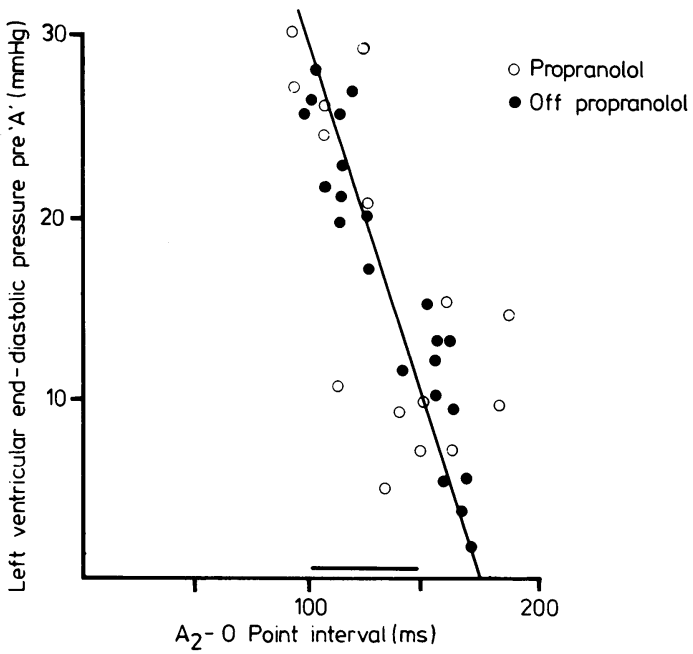

Fig. 4 Relation between left ventricular end-diastolic pressure and the interval A2-0 point in patients on or off beta-blocking drugs. The $95 \%$ confidence limits normal for A2-0 point interval are indicated by the horizontal bar.

time fell below $40 \mathrm{~ms}$, the height of the $\mathrm{f}$ wave increased strikingly.

\section{EFFECTS OF GLYCERYL TRINITRATE AND \\ ISOMETRIC EXERCISE}

(1) Heart rate, arterial pressure, and cavity dimensions These are given in detail in Fig. 6. Glyceryl trinitrate caused a significant increase in heart rate and a reduction in systolic and diastolic blood pressure. Cavity dimensions also fell at end-systole and end-diastole. Isometric stress caused increases in heart rate, and in systolic and diastolic blood pressures. In contrast to glyceryl trinitrate, cavity dimensions increased at end-diastole and end-systole. There were no significant differences in the behaviour of any of these $\circ$ variables between the normal subjects and those with coronary artery disease, whether contraction pattern was co-ordinate or incoordinate.

\section{(2) Diastolic time intervals}

In patients with ischaemic heart disease, glyceryl N్ trinitrate caused significant prolongation of isovolumic relaxation by $23 \mathrm{~ms}$, which was highly significant $(p<0.01)$. Prolongation of isovolumic relaxation time was greatest in patients with high filling pressures but $\stackrel{\oplus}{\rightarrow}$ also occurred in patients with normal filling pressures.

A2 to the 0 point was also prolonged by $12 \mathrm{~ms}$ $(p<0.01)$. This was less than the delay in mitral valve $\stackrel{\mathbb{D}}{\odot}$ opening, so that the interval mitral valve opening to $\mathbb{\mathbb { D }}$ the 0 point was significantly shortened. At the same time, the 0-f interval was prolonged by $20 \mathrm{~ms}$ 


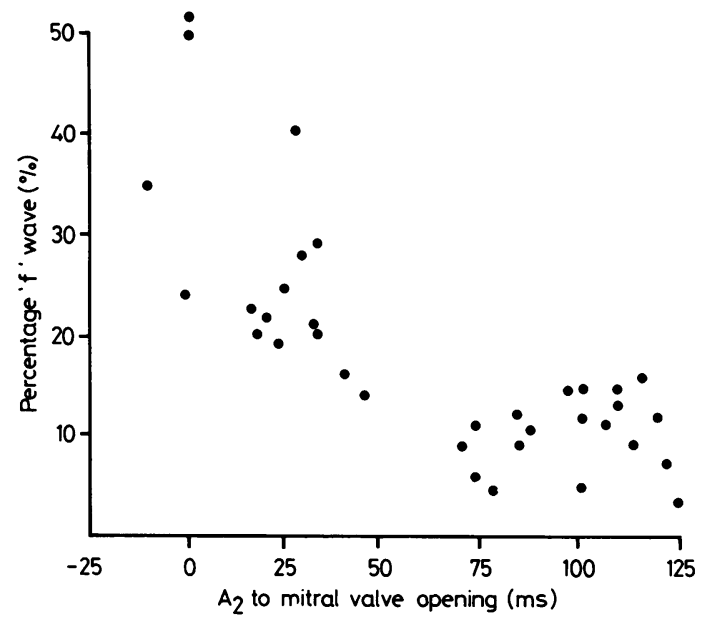

Fig. 5 Relation between isovolumic relaxation time and the relative height of the $f$ wave $(\% f)$.

$(p<0.01)$, and the relative amplitude of the $f$ wave fell by $23 \%$ of the initial value.

Isometric stress caused isovolumic relaxation time to shorten by $17 \mathrm{~ms}(\mathrm{p}<0.01)$ in the patients. A2-0 point and $0-f$ intervals also tended to shorten, though the sample size was small for these measurements, because of technical difficulties in obtaining apexcardiograms during exercise. There were no changes in A2 - MVO or A2 - 0 point intervals in normal subjects in response to glyceryl trinitrate or isometric handgrip.

(3) Relation of changes in diastolic time intervals to resting contraction pattern

Changes in diastolic time intervals correlated with resting contraction pattern. In 15 patients with ischaemic heart disease and a normal left ventricular end-diastolic pressure, nine responded to glyceryl trinitrate administration with changes in isovolumic relaxation time; of these, seven had a synchronous contraction pattern and two an asynchronous pattern. All of the six patients who failed to respond to glyceryl trinitrate had an asynchronous contraction pattern $(p<0.01)$. Of 11 patients who underwent isometric stress testing, seven had shortening of isovolumic relaxation time, and in six of these contraction was synchronous. Of the four patients not responding to isometric handgrip, all four were asynchronous $(\mathrm{p}<0.02)$.

\section{(4) Contraction and relaxation pattern}

Glyceryl trinitrate did not affect peak shortening, but there was a significant increase in dimension change during the time of inscription of the downstroke of the apexcardiogram $(\mathrm{p}<0.05)$ from $37 \pm 20 \%$ to $48 \pm 30 \%$, while mitral valve opening was further delayed with respect to minimum dimension $(p<0.05)$ by $23 \pm 4 \mathrm{~ms}$. Isometric stress caused peak shortening to fall significantly $(p<0.05)$. It did not alter left ventricular dimensions during either the upstroke or the downstroke of the apexcardiogram but led to a reduction in the delay of mitral valve opening with respect to minimum dimension by $30 \pm 5 \mathrm{~ms}(\mathrm{p}<0.01)$.

\section{(5) Effect of beta-blocking drugs}

Of the 55 patients in this study, 16 were on long term beta-blockade. There was no correlation between treatment and resting contraction pattern (whether co-ordinate or incoordinate), or between end-diastolic pressure and diastolic time intervals except that patients on beta-blockers showed a poorer correlation between left ventricular end-diastolic pressure and A2-0 point interval. No aspect of the response to glyceryl trinitrate or isometric stress was significantly altered in patients taking beta-blocking drugs.

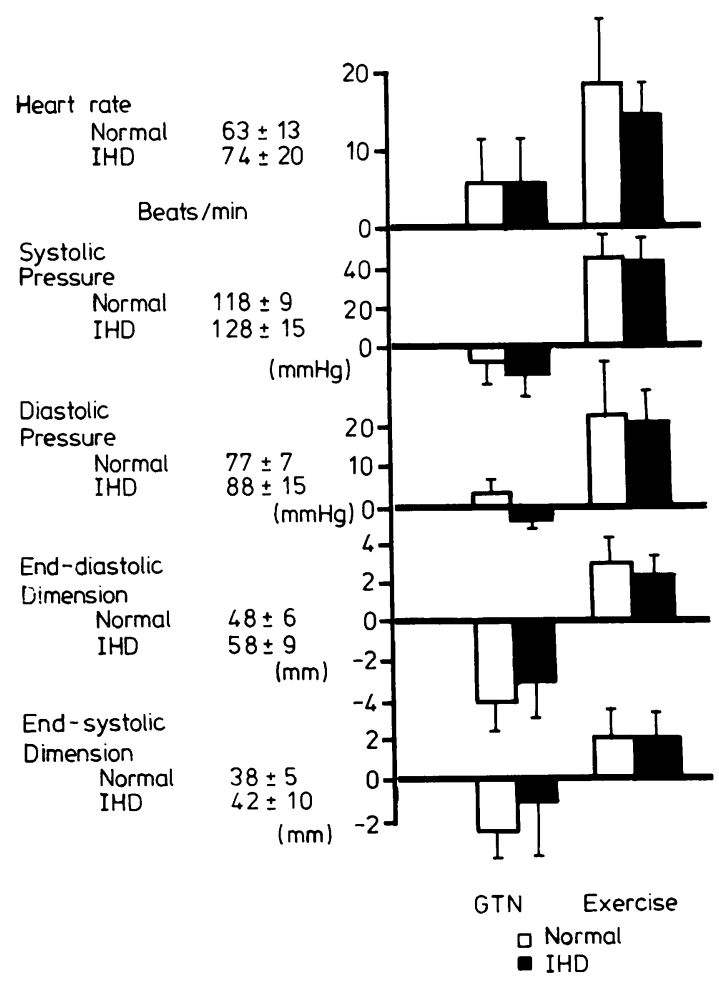

Fig. 6 Changes in heart rate, systolic and diastolic blood pressure, and end-systolic and diastolic dimension with glyceryl trinitrate $(G T N)$ and isometric stress. 


\section{Discussion}

Non-invasive methods can be used to define a series of events during early diastole, including A2 from the phonocardiogram, and the 0 and $f$ points of the apexcardiogram. None of these coincides precisely with measurements obtained invasively. Thus A2 bears no fixed relation to the timing of peak negative $\mathrm{dP} / \mathrm{dt}$, mitral valve opening may precede the cross-over of atrial and ventricular pressures, ${ }^{3}$ the 0 point of the apexcardiogram, though close to the nadir of the pressure pulse, is not synchronous with it, ${ }^{4}$ and the $f$ wave, corresponding to the third heart sound, has no invasive counterpart. These non-invasive measurements are reproducible, however, and represent a series of events of considerable physiological or pathological significance, so that their study becomes a legitimate object of inquiry in its own right.

Mean values for isovolumic relaxation time reported here agree with those previously reported. ${ }^{5}$ The increased scatter of values about the mean, seen in patients with ischaemic heart disease, was unrelated to aortic pressures, but could be largely explained on the basis of left ventricular end-diastolic pressure, to which isovolumic relaxation time was strongly and negatively correlated. In the group of patients in whom end-diastolic pressure was normal, isovolumic relaxation was very significantly prolonged to nearly $100 \mathrm{~ms}$, a value almost identical to that previously reported in patients with left ventricular hypertrophy. ${ }^{5}$ The mechanism underlying this prolongation was not clear. It was not the result of concomitant left ventricular hypertrophy, nor could it be related to incoordinate wall motion, either during isovolumic contraction or isovolumic relaxation. Prolongation of isovolumic relaxation time thus appears to reflect some other aspect to left ventricular involvement in ischaemic heart disease, which we are, at present, unable to identify.

A raised end-diastolic pressure affected other diastolic events. It was associated with an increase in the prematurity of mitral valve opening with respect to the 0 point, and a decrease in the time interval from A2 to the 0 point. This latter interval has been studied in the past as isovolumic relaxation, ${ }^{6}$ but can more usefully be regarded as a measure of the time taken by the left ventricular pressure to fall to its minimum value at the end of ejection. Like isovolumic relaxation, it was longer than normal in patients with ischaemic heart disease, and was also strongly and inversely correlated with end-diastolic pressure. The timing and relative amplitude of the $f$ wave were also related both to the duration of isovolumic relaxation time and to end-diastolic pressure, a high enddiastolic pressure being associated with an earlier $f$ wave of increased relative amplitude. No such relation could be shown between these values and either $c$ shortening fraction or peak shortening, which reflect other aspects of left ventricular function. It is apparent, therefore, that these diastolic time intervals do not vary randomly, but that characteristic patterns are seen in patients with ischaemic heart disease, dependent on end-diastolic pressure.

In order to investigate the apparent stability of $\approx$ these patterns further, we attempted to perturb left $\vec{\circ}$ ventricular function by subjecting it to the effects of glyceryl trinitrate administration or isometric stress. Both interventions caused changes in arterial pressure and cavity size that have previously been described. ${ }^{7}$ Neither intervention had any additional effect during early diastole in the normal subjects. In patients with $\omega$ ischaemic heart disease, however, well defined of changes were observed. Glyceryl trinitrate prolonged isovolumic relaxation time if it was initially short, delayed the 0 point of the apexcardiogram with respect to $A 2$, increased the interval between the 0 point and the $f$ wave, and reduced its relative amp- $\overline{3}$ litude. Conversely, isometric stress shortened isovolumic relaxation and the intervals $\mathrm{A} 2-0$ point and 0 point $-f$ wave. Thus, the interrelations between diastolic events were maintained, the changes after glyceryl trinitrate being similar to those expected with a reduction in end-diastolic pressure, and those after isometric stress with an increase. Since the study was performed non-invasively, end-diastolic pressure was not measured directly, though similar changes are well documented. ${ }^{8}$ It would clearly be of interest to do so, since an alternative possibility is that changes in diastolic time intervals and end-diastolic pressure both reflect some more fundamental modification in left ventricular properties brought about by these interventions.

Simultaneous M-mode echocardiograms of the left $\frac{3}{3}$ ventricular cavity and apex-or phonocardiograms can be used to detect disturbances in contraction pattern. Normally changes in transverse dimension are small 0 during the time of inscription of the upstroke of the apexcardiogram, which approximates to isovolumic $\frac{7}{0}$ contraction, and during isovolumic relaxation. An asynchronous contraction pattern is shown by $\stackrel{\sim}{\sigma}$ significant dimension changes during isovolumic con- $N$ traction or isovolumic relaxation, representing N changes in cavity shape at a time in the cardiac cycle $\sigma$ when ventricular volume is constant, and by minimum cavity dimension occurring early, so that it comes to coincide with A2. Analysis of the results in $\stackrel{0}{\rightarrow}$ individual patients in the present study showed that the effects of both interventions were determined by 0 basal contraction pattern. Only when contraction pat- $\mathbb{D}$ tern was synchronous, did isovolumic relaxation time $\overrightarrow{\mathbb{D}}$ alter with glyceryl trinitrate or handgrip. When it was $\frac{\varrho}{\sigma}$ asynchronous, the extent of abnormal dimension 
changes occurring during isovolumic relaxation was modified though their duration remained unchanged. The effects of glyceryl trinitrate are thus similar to those previously shown for propranolol, 19 both in their nature and in their sensitivity to basal contraction pattern, though they differ because, with the later drug, they occurred when contraction pattern was asynchronous rather than synchronous.

This consistent pattern seems to require explanation. The changes cannot be explained on the basis of heart rate, and are the exact opposite of what would be predicted from alterations in arterial pressure. The reduction in isovolumic relaxation time with increasing end-diastolic pressure is likely to be, in part, a direct effect of the concomitant rise in left atrial pressure, as has previously been noted in mitral stenosis, and more recently in a variety of other types of heart disease. ${ }^{10}$ The lack of sensitivity of isovolumic relaxation time to aortic pressure could be explained on the basis of the close time relation between peak negative $\mathrm{dP} / \mathrm{dt}$ and $\mathrm{A} 2$, so that left ventricular pressure is falling more rapidly when the valve closes than when the mitral valve opens. The basis for the changes in the apexcardiogram is less obvious. The changes were not seen in normal subjects, and are independent of the . timing of mitral valve opening, so they bear no necessary relation to left atrial pressure. One possibility is that they represent a clinical manifestation of the load dependence of relaxation, ${ }^{11}$ the rate of relaxation, and therefore the rate of pressure fall, altering with changes in end-systolic dimension. Against this idea is their failure to occur in normal subjects in spite of virtually indentical dimension changes. In addition, changes were seen in the $0-\mathrm{f}$ interval, as well as in the relative amplitude of the $f$ wave itself. These events occur well beyond the end of relaxation, suggesting that the passive behaviour of the ventricle had also been modified. Of the passive time-related properties of a fluid-containing ventricle with elastic walls, the most relevant to the present observations appear to be simple mechanical oscillations, whose periodicity and degree of damping were increased by glyceryl trinitrate and decreased by isometric stress. The study of such oscillations in the heart is extremely complex, and indeed the problem of predicting the natural frequency of a fluid filled ventricle of irregular shape has not been solved completely. In general terms it is possible to state that the natural frequency is proportional to the mass of the system and the stiffness of the walls, and tends to be greater when the cavity is spherical. There is no evidence that glyceryl trinitrate or propranolol caused an increase in end-systolic cavity size or a change in shape, but their effect on diastolic events might be explained by their altering the passive stiffness of the ventricular walls. It may be significant that such an effect on pressure volume relations has been observed for both drugs later in diastole. ${ }^{8}{ }^{12}$ The presence of such oscillations would have clear implications both for interpreting diastolic events, and for understanding the relation between the time course of relaxation in the myocardium and the cavity pressure trace, so that further investigation of their possible presence seems justified.

These theoretical considerations apart, the observations reported here have clinical significance. They indicate that prolongation of isovolumic relaxation occurs consistently in patients with coronary artery disease, and that it reflects some so far unidentified aspect of left ventricular involvement in this condition. A short isovolumic relaxation time, in the absence of mitral valve disease, is good evidence of a raised end-diastolic pressure. We have used this observation to monitor the long term effects of nitrates in patients with severe left ventricular disease. The occurrence of a group of changes involving several independent variables reduces the uncertainty with which observations can be made, and increases the conviction of abnormalities detected in individual patients. The repreducible nature of these noninvasive measurements adds weight to the contention that they have become useful tools in the study of normal and abnormal diastolic events in man.

\section{References}

1 von Bibra $H$, Gibson DG, Nityanandan $K$. Effects of propranolol on left ventricular wall movement in patients with ischaemic heart disease (abstract). Br Heart $\mathcal{F}$ 1979; 43: 231-2.

2 Gibson DG, Brown D. Measurement of instantaneous left ventricular dimension and filling rate in man, using echocardiography. Br Heart $\mathcal{F}$ 1973; 35: 1141-9.

3 Tsakiris AG, Gordon DA, Padiyar R, Fréchette D. Relation of mitral valve opening and closure to left atrial and ventricular pressures in the intact dog. Am $\mathcal{F}$ Physiol 1978; 234: H146-51.

4 Willems JL, de Geest H, Kesteloot $\mathrm{H}$. On the value of apex cardiography for timing intracardiac events. Am $\mathcal{F}$ Cardiol 1971; 28: 59-66.

5 Chen W, Gibson D. Relation of isovolumic relaxation to left ventricular wall movement in man. Br Heart $\mathcal{f} 1979$; 42: 51-6.

6 Benchimol A, Ellis JG. A study of the period of isovolumic relaxation in normal subjects and patients with heart disease. Am $\mathcal{F}$ Cardiol 1964; 19: 196-206.

7 Redwood DR, Henry WL, Epstein SE. Evaluation of the ability of echocardiography to measure acute alterations in left ventricular volume. Circulation 1974; 50: 901-4.

8 Mann T, Goldberg S, Mudge GH Jr, Grossman W. Factors contributing to altered left ventricular diastolic properties during angina pectoris. Circulation 1979; 59: 14-20.

9 Gibson DG, Wong P. Effect of sublingual nitroglycerin on left ventricular wall movement in patients with coro- 
nary artery disease: comparison with propranolol and saphenous bypass grafting. Nouv Presse Med 1980; 9: 2393-8.

10 Palomo AR, Quinones MA, Waggoner AD, Kumpuris AG, Miller RR. Echo-phonocardiographic determination of left atrial and left ventricular filling pressures with and without mitral stenosis. Circulation 1980; 61: 1043-7.

11 Brutsaert DL, De Clerck NM, Goethals MA, Housmans PR. Relaxation of ventricular cardiac muscle. F Physiol (Lond) 1978; 283: 469-80.
12 Coltart DJ, Alderman EL, Robison SC, Harrison DC? Effect of propranolol on left ventricular function $\vec{F}$ sequential wall motion and diastolic pressure-volume relation in man. $\mathrm{Br}$ Heart $\mathcal{f}$ 1975; 37: 357-64.

Requests for reprints to Dr PJ Oldershaw, Cardiac® Department, St George's Hospital, Blackshaw Road London SW17 0RE. 